\title{
An Improved Method of Photographic Measures of Double Stars
}

\author{
D. Rodriguez ${ }^{1}$, J.A. Bernedo ${ }^{1,2}$ and D. Valls-Gabaud ${ }^{1,3}$
}

1 Agrupación Astronómica de Madrid, Apartado 1039, E-28080 Madrid, Spain

2 Planetario de Madrid, Parque Tierno Galván, E-28045 Madrid, Spain

3 Institut d'Astrophysique de Paris, 98 bis, Bld. Arago, F-75014 Paris, France

Summary. We present a new technique in double star photography leading to high accuracy measurements in amateur-size instruments. The trailing method has been improved by the use of a chopper that cuts the trail into a number of segments. The chopper period is accurately known, so it is the length of each segment on the plate. In this way the scale factor is measured in each exposure. Fine-grain films, such as hyper-sensitized Kodak 2415, are used and all measurements are performed at the microscope. The same technique may also be applied to CCD cameras. A statistical analysis of errors shows that $\Delta \theta \leq 30^{\prime}$ and $\Delta \rho / \rho \approx 0.01$ for most binaries with $\rho \geq 3^{\prime \prime}$. This method has been applied to professional-amateur joint programs dealing with multiple systems with variable components and a survey of poorly-observed wide binaries.

\section{Introduction}

We started this work to measure the parameters of a series of wide double stars of which the most recent data found in catalogues were obtained in years around 1910 , and remained practically unobserved since then. The second project was to detect possible changes in systems with variable components. From the amateur's point of view, the means to carry out this work are modest, even with the newest techniques [Ref. S), so it was fundamental to design a new method to insure the maximum accuracy possible, and above all, a reliable way of accounting for the errors in each stage of the measuring process. This point is particularly important for two reasons : (i) In the computation of orbital elements, the róle played by the uncertainties in each measure is crucial [Refs. 2,11], (ii) The knowledge of errors could help to understand some trends that frequently appear in photographic determinations of P.A. and separation (e.g. the Kostinsky effect [Ref. 5], and the dependence of the P.A. error on separation [Refs. 9,12]).

A second part of this work is the study of the same stars with small professional instruments (Observatoire de Paris-Meudon), in order to establish a comparison between results and check for possible systematic errors and to further improve the whole process. The report of this part will be described elsewhere.

\section{The Method}

2.1 Photographic recording. The use of visual observing systems were discarded due to the high cost of the equipment involved for the desired accuracy, or to the high intrinsic errors expected. For instance, new filar amateur micrometers [Ref. 10$]$ provide $\Delta \theta \approx 20^{\prime}$ and $\Delta \rho \approx 0 .{ }^{\prime} 5$, while the double image micrometer [Ref. S] gives slightly better results but is limited to bright pairs. Moreover, in most micrometer techniques, but the diffraction grating one $[R e f s .3,6]$, the calibration remains difficult if high accuracy is desired.

We used a film hyper-sensitizing technique, developped at the Agrupación Astronómica de Madrid for 4 years and which has shown a very high performance. We treated the micrographic film Kodak 2415 with pure hydrogen hyper-sensitizing process at a pressure of $2 \mathrm{~kg} \mathrm{~cm}$ and kept at $36^{\circ} \mathrm{C}$ during 48 hours, resulting in a very high resolution, 1000 ASA sensitivity film.

On this film the following must be recorded in order to use our technique: (i) The images of both components (with the proper telescope tracking), (ii) A trail (without tracking), to define the East-West direction (essential for position angle measurements), and (iii) The scale factor: a measurement with which to convert all linear dimensions into angular quantities. 
2.2 Measure of time. The scale factor is the key point of this study. Most observers take "fixed" pairs or the Pleyades to calibrate their measurements $|R e / s .1,3,1,6|$. This technique is right only if there is a complete sample of well-measured, fixed pairs for all separations and magnitudes. It can be useful to take some standards from high-quality measures [i.e. Ref. 7], but there are very few of these at magnitudes fainter than 8.

Taking into account that it will affect all measurements, its accurate determination is essential. We tried first electromechanic shutters (EMS) driven by electronic clocks, but their dispersion were too high for our purposes. We finally came out to a solution which avoided these problems : a rotating shutter driven by a synchronous motor. Quite differently from others methods, we place the shutter in front of the telescope aperture in a way that the light path to the primary mirror/objetive is interrupted. This configuration has the disadvantage that not all the incoming light is cut at the same time, so the shutter dimension in the direction of motion has to be comparable with the telescope diameter. In this way we obtained images like the shown in Figure 1 for Cor Caroli $(\alpha \mathrm{CVn})$. The extinction of the trail is not instantaneous, but occurs in a short interval as it is the case for the reappearance of the trail. To prevent this phenomenon from impairing the measurements, it was necessary to choose the right relationship between shutter dimensions and the distance of its rotation axis to the centre of the telescope aperture, as shown in Figure 2. We found that the best trail is obtained when the segment completely dissappears, leaving $1 / 10$ to $1 / 5$ of a segment before a new one (Fig. 2.2).

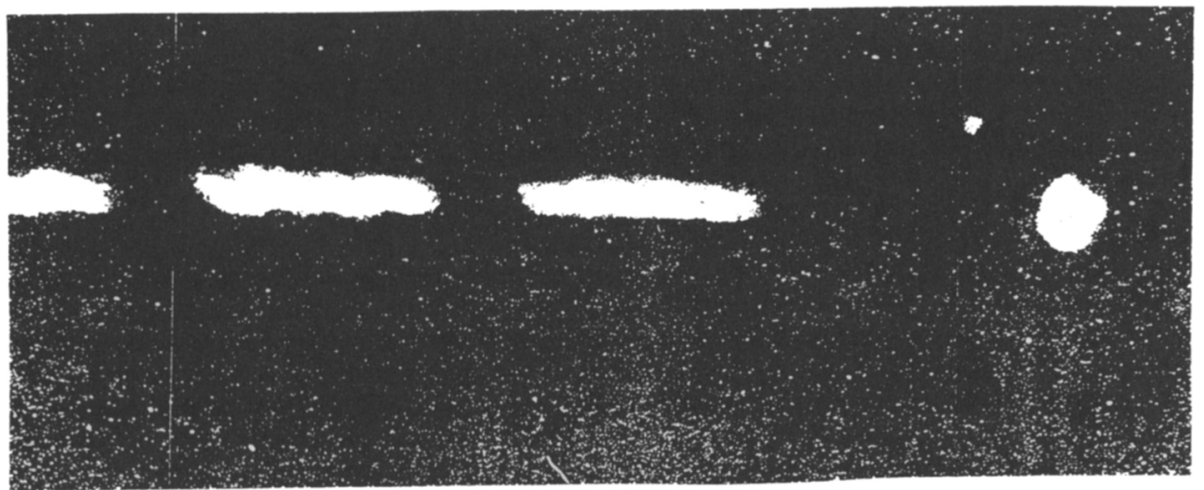

Figure 1. Positive copy. General aspect. $\alpha$ CVn system.

The procedure is the following: (1) We record the images of both components with telescope tracking and the right exposure time (see [Refs. 3,4] for an analysis of $t_{\text {exp }}$ as a function of the magnitude). (2) We stop the tracking, switch the synchro motor on and let the image drift across the frame as long as possible. This second step records at the same time the $\mathrm{E}-\mathrm{W}$ direction and the time scale.

A precision in the chopping of the trail of $\pm 0.05 \mathrm{~Hz}(0.1 \%)$ with a $95 \%$ probability that the error is below $\pm 0.03 \mathrm{~Hz}(0.06 \%$, adopted value) is obtained.

In relation to the speed of the motor, we looked for a compromise between two factors. On one side, the greater the number of segments on the trail, the smaller mean accuracy in the segment measure. On the other, few segments imply the risk that, having to skip the first segment wich corresponds to the starting of the motor and the last one (uncompleteness, deformations due to proximity to the edge of the frame) we count with only very few segments, increasing errors accordingly (Fig. 4). The final speed chosen was 1. cycle every 4 seconds (i.e., 0.250 cycles/sec).

The measurement of the scale factor is made by taking into account several segments (all possible but the first and last ones). This error is reduced just because the time error is known, and the error due to border estimation (bigger) is distributed over a long distance. The measurements were performed with a Nikon Metaphot microscope, with a mean precision in the micrometric movements of $\pm 0.005 \mathrm{~mm}$ at $50 \mathrm{X}$. Typical values of border estimation errors are $\pm 0.002 \mathrm{~mm}$ on a $35 \mathrm{~mm}$ frame. We also used a slide projector and took measures with a mi- 


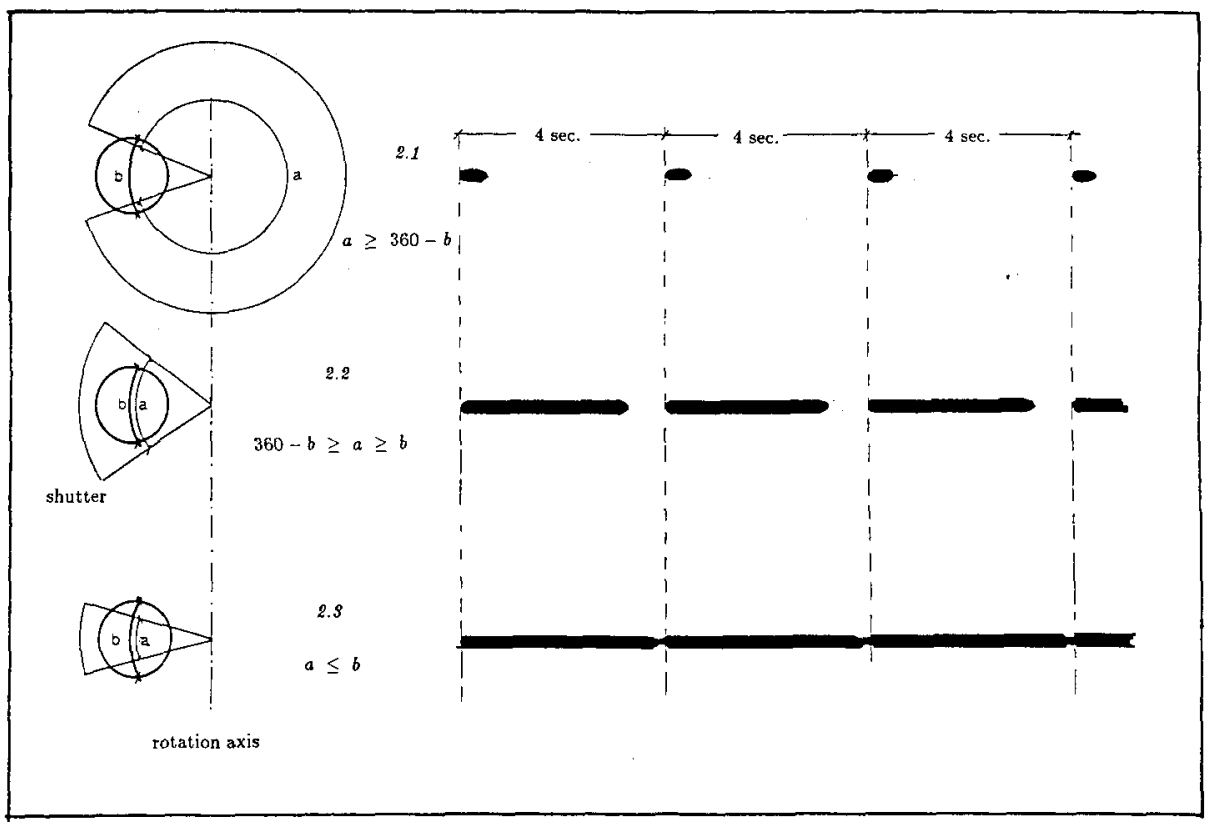

Figure 2. Influence of shutter-aperture relationship on the trace.

crometer or a good calliper. The error suffered in this process was 0.20 to $0.25 \%$. The final error for the scale factor measurement is typically $0.31 \%$.

2.3 Measurement of separation. At this stage, two different techniques have been employed (Table I), as illustrated in Fig. 5. A simple inspection indicates that the latest gives a higher error, which is consistent with the fact that it involves a quadratic operation. Typical errors at this point are $0.6 \%$ (direct method) and $0.9 \%$ (coordinate method). The ideal measurement consists in determining the photocentre of each component (via a microdensitometer or by image processing). The critical parameter at this stage is the exposure time (over-, under- exposition, seeing and turbulence effects, see Ref. 5).

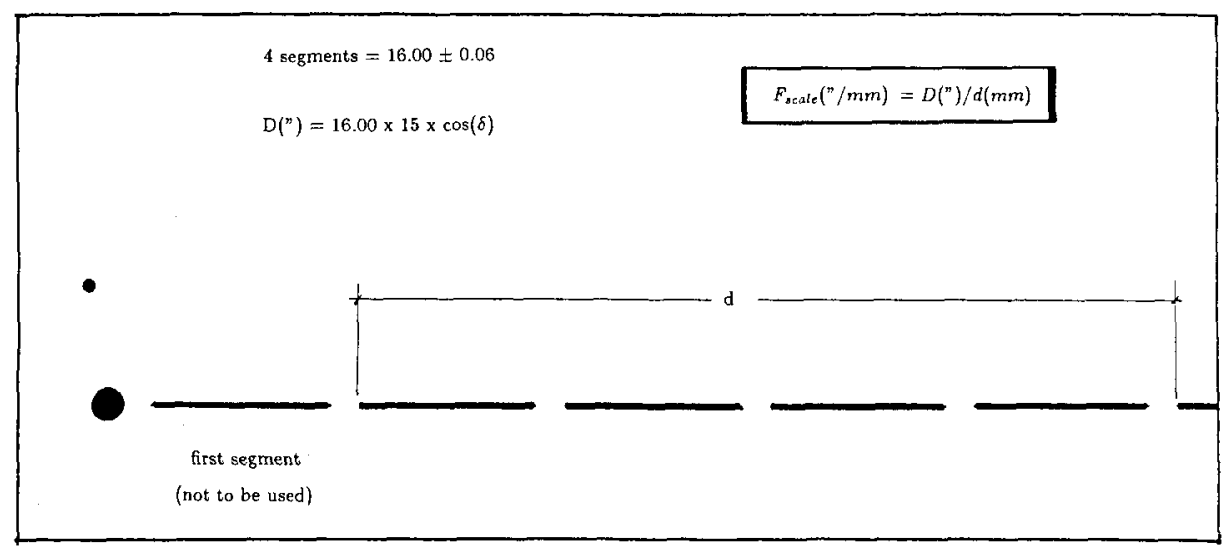

Figure 4. Measure of the scale factor. Example. 
TABLE I

\begin{tabular}{ccccccccc}
\hline System & S & Esf $\%$ & $\rho_{\text {dir }}(")$ & E.\% & $\rho_{\text {coord }}(")$ & E.\% & $\theta_{\text {arc.tan }}\left({ }^{\circ}\right)$ & $\theta_{\text {arc.cos }}\left({ }^{\circ}\right)$ \\
\hline$\beta$ Cyg & 6 & 0.31 & $34.61 \pm 0.35$ & 1.01 & $\mathbf{3 4 . 5 4 \pm 0 . 4 5}$ & 1.32 & $53.48 \pm 0.54$ & $53.33 \pm 1.21$ \\
17 Boo & 1 & 0.49 & $13.65 \pm 0.21$ & 1.59 & $13.76 \pm 0.27$ & 2.02 & $235.71 \pm 0.45$ & $236.37 \pm 1.72$ \\
S Lyr & 7 & 0.37 & $44.12 \pm 0.41$ & 0.94 & $43.99 \pm 0.52$ & 1.12 & $150.14 \pm 0.49$ & $150.24 \pm 0.82$ \\
$\alpha$ CVn & 3 & 0.38 & $19.25 \pm 0.22$ & 1.17 & $19.06 \pm 0.28$ & 1.50 & $227.54 \pm 0.21$ & $226.92 \pm 0.68$ \\
$\alpha$ Lib & 4 & 0.24 & $230.41 \pm 0.40$ & 0.44 & $232.59 \pm 1.03$ & 0.44 & $313.64 \pm 0.10$ & $313.64 \pm 0.18$ \\
\hline
\end{tabular}

$\mathrm{S}$ is the number of segments, Esf gives the error on the scale factor, and $\mathrm{E}$ on the preceding $\rho$.

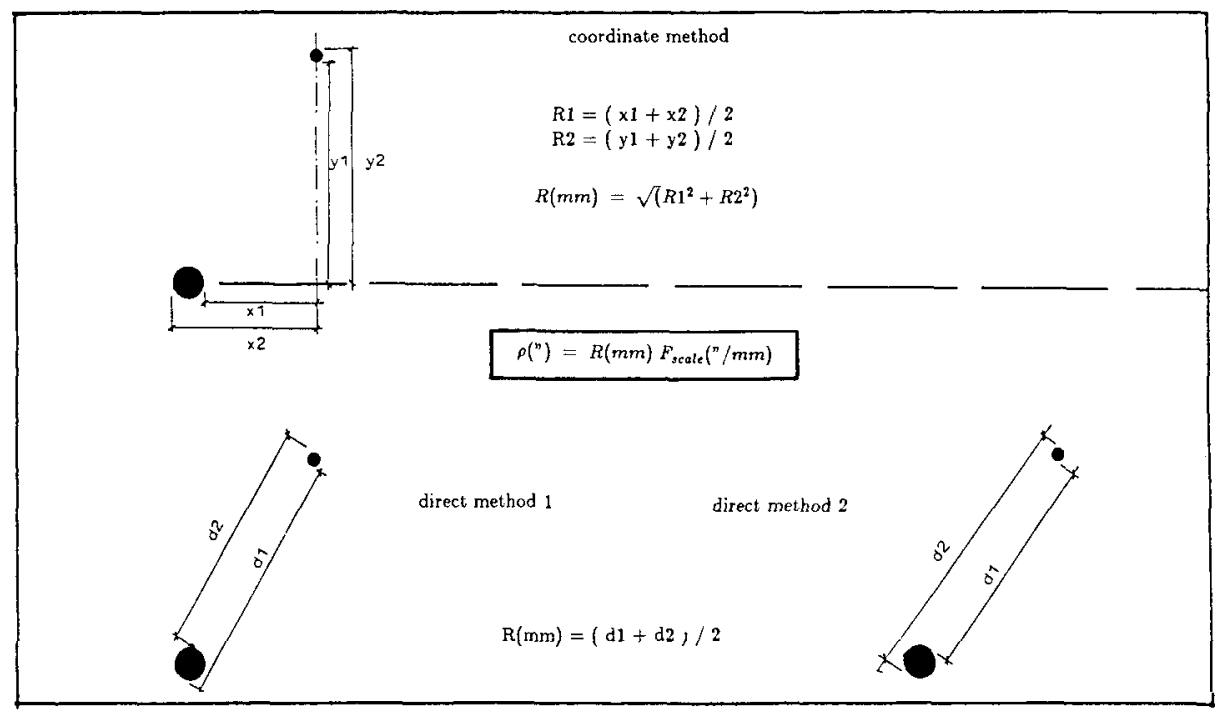

Figure 5. Measure of separation.

2.4 Measurement of position angle. Two different coordinate techniques may be used, one based on the use of the arc tangent and the other on the arc cosine (Fig. 6). For them, typical errors obtained were $18^{\prime}$ for the first one and $50^{\prime}$ for the second.

2.5 Total error. The total error has been calculated on the basis of the worst possible occurence. For the scale factor, the error is found to be around 0.24 to $0.49 \%$, while for the separation, 0.46 to $2.08 \%$ (coordinate method), and 0.40 to $1.87 \%$ (direct method) are obtained. The errors for the arc tan technique are found to be 6' to $54^{\prime}$, while the arc cosine technique gives 11' to 78' respectively. This dispersion of errors is due to their dependence on a number of factors as the border definition and background light.

\section{The practice}

In practice, this work has posed two main problems. The worst one was to record the chopped trail with a good definition. In fact, this problem is frequently found when using the trail method. For example, the Torino group $[R e f s .9,12]$ takes series of some 20 contiguous exposures, followed by the orientation trail. In some cases, the trail is too faint and they are unable to measure the position angle. The second problem was the limiting magnitude. The image of a secondary is well recorded in exposure but is limited by the magnitude of the primary to certain values, in order to avoid large, spread images which increase the errors. The first case imposed a limit magnitude for the primary of 4.6 , and in the second, a 6.7 limiting magnitude for the secondary. 
TABLE II

\begin{tabular}{cccccc}
\hline System & $\rho_{\text {cat }}$ & Year & $\theta_{\text {cat }}$ & $\rho_{\text {abs }}$ & $\theta_{\text {obs }}$ \\
\hline$\beta$ Cyg & 34.4 & 1967 & 54.1 & $34.6 \pm 0.3$ & $53.5 \pm 0.5$ \\
$17 \mathrm{Boo}$ & 13.5 & 1973 & 236.2 & $13.6 \pm 0.2$ & $235.7 \pm 0.4$ \\
S Lyr & 43.7 & 1955 & 150.0 & $44.1 \pm 0.4$ & $150.1 \pm 0.5$ \\
$\alpha \mathrm{CVn}$ & 19.3 & 1974 & 228.4 & $19.2 \pm 0.2$ & $227.5 \pm 0.2$ \\
$\alpha \mathrm{Lib}$ & 231. & 1913 & 314. & $230.4 \pm 0.9$ & $313.6 \pm 0.1$
\end{tabular}

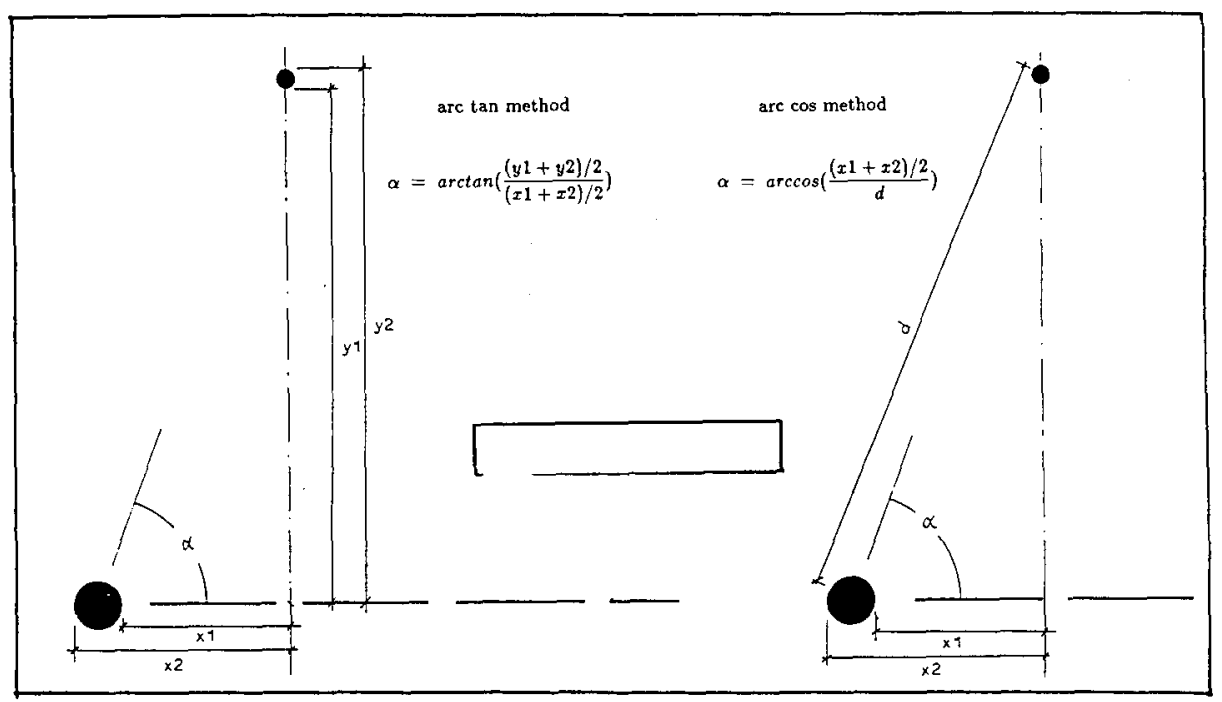

Figure 6. Measure of position angle.

We follow the simple scheme suggested by J. Dommanget $[$ Ref. 2 $\mid$ for the data analysis. The final results are given as $\langle\rho\rangle \pm \epsilon_{\langle\rho\rangle}$ and $\langle\theta\rangle \pm \epsilon_{\langle\theta\rangle}$ where \langle\rangle means a weighted average, and $\epsilon$ is the final error on the average value. The results that follow were obtained with a refractor of $90 \mathrm{~mm}, \mathrm{f} / 15$, and a newtonian reflector of $200 \mathrm{~mm}, \mathrm{f} / 5$, from a site near Villaviciosa de Odón, Madrid. As shown in Table II, the errors obtained are remarkably smaller than the typical visual ones $[$ e.g. Refs. 8,10], and the results agree with other data $[R e f s .6,19 \mid$. Recent photographic measures of $\beta$ Cygni and $\alpha \mathrm{CVn}[R e f$. 4] give very similar results, even if the 0.1 " error claimed in that paper seems to be quite optimistic.

\section{Conclusions}

The present work attempts to show that the use of a simple technique, simultaneously recording the images of components, the $\mathrm{E}-\mathrm{W}$ direction and an accurate measure of time, avoids and reduces systematic errors in great proportion, besides the aditional point of keeping a record of the data.

The use of a rotating shutter has proven fundamental in the reduction of the scale factor and total errors, and its easy construction guarantees its availability to amateurs, which in this way may obtain valuable data including the accuracy range of their measures, essential for orbit determinations. The method slightly improves the accuracy of P.A., but results are not so good as for the separation. Image processing of the corresponding CCD frames reduces these errors by factors 3 to 5 , depending on the signal-to-noise ratio. A rotating shutter as the proposed one remains to be developped if mounted near the focal plane of the camera. The use of this system with wider aperture telescopes will accordingly increase the limiting magnitude and image definition and will be of great assistance to the systematic measure of visual binaries.

This is an highly abridged version of our work. A complete version is available upon request. 


\section{References}

(1) P. Couteau (1978). L'observation des étoiles doubles visuelles, pp. 67-69. Flammarion.

2] J. Dommanget (1959). Ann. Obs. Roy. Belgique (Série 3) $8:$ 89-93.

3] P. Durand (1987) in Astronomie-Le guide de l'observateur. Vol. 2, pp.669-722. S.A.P.

[4] P. Durand, D. Simier (1987). L'Astronomie $101: 265-276$.

[5] W.D. Heintz (1978). Double stars. pp. 26-31. Reidel.

6) K.G. Jones (1979). Webb Society Handbook. Volume 1. Double stars. pp. 49-58.

[7] F.J. Josties , R.S. Harrington (1984). Astrophys. J. Suppl. Ser. 54 : 103-113.

[8] D.J. MacConnell (1978). Astron. J. 83 : 436-437, and (1984) Astron. J. $89: 876-878$.

[9] R. Morbidelli, R. Pannunzio (1987). Astron. Astrophys. Suppl. Ser. 68 : 481-492.

[10] J.A. Soldevilla (1985). Astrum 60 : 15-16.

[11] E. Soulié (1986). Astron. Astrophys. 164 : 408-414.

[12] R. Pannunzio, R. Morbidelli (1984). Astron. Astrophys. Suppl. Ser. 55 : 451-454. 